Pacific Journal of Mathematics

INTEGRAL REPRESENTATION FORMULAS ON ANALYTIC
VARIETIES 


\title{
INTEGRAL REPRESENTATION FORMULAS ON ANALYTIC VARIETIES
}

\author{
Telemachos E. Hatziafratis
}

\begin{abstract}
Integral representation formulas for holomorphic functions on analytic subvarieties of domains of $\mathrm{C}^{n}$ are derived. These formulas generalize the Cauchy-Fantappiè formula and the Weil formula for analytic polyhedra. The kernels we obtain are explicitly defined.
\end{abstract}

Introduction. In recent years integral formulas and their applications have attracted a lot of attention in several complex variables; see for example $[4,5,7,8,9,10]$ and the most relevant to our work papers of Stout [15], Palm [11] and Henkin and Leiterer [6].

In this paper we develop analogues of Cauchy-Fantappiè Kernels for analytic subvarieties of domains of $\mathbf{C}^{n}$. First let us recall the CauchyFantappiè formula. Let $D \subset \mathbf{C}^{n}$ be a bounded domain with smooth boundary and let $\gamma:(\partial D) \times D \rightarrow \mathrm{C}^{n}$ be a smooth function so that

(1) $(\zeta-z, \gamma(\zeta, z))=: \sum_{j=1}^{n}\left(\zeta_{j}-z_{j}\right) \gamma_{j}(\zeta, z) \neq 0$

$$
\text { for }(\zeta, z) \in(\partial D) \times D \text {. }
$$

Then the Cauchy-Fantappiè formula associated to $\gamma$ is the following:

For $f \in \mathcal{O}(D) \cap C(\bar{D})$ and $z \in D$ we have:

(2) $f(z)=(-1)^{n(n-1) / 2+1} \frac{(n-1) !}{(2 \pi i)^{n}} \int_{\zeta \in \partial D} f(\zeta) \frac{\omega^{\prime}(\gamma(\zeta, z)) \wedge \omega(\zeta)}{(\zeta-z, \gamma(\zeta, z))^{n}}$

where

$$
\omega^{\prime}(\gamma(\zeta, z))=: \sum_{j=1}^{n}(-1)^{\prime} \gamma, \bigwedge_{k \neq j} \bar{\partial}_{\zeta} \gamma_{k} \quad \text { and } \omega(\zeta)=: d \zeta_{1} \wedge \cdots \wedge d \zeta_{n} .
$$

In §I we give a generalization of the formula (2) from the domain $D$ to analytic subvarieties of $D$. More precisely we consider a bounded pseudoconvex domain $D$ with smooth boundary and $m(m<n)$ holomorphic functions $h_{1}, \ldots, h_{m}$ in $U$ where $U$ is a domain containing the closure $\bar{D}$ of $D$. We set

$$
\begin{gathered}
V=:\left\{z \in U: h_{1}(z)=\cdots=h_{m}(z)=0\right\}, \\
M=: V \cap D \text { and } \partial M=: V \cap(\partial D) .
\end{gathered}
$$


Our assumptions are that the variety $V$ should have no singular point on $\partial M$ and that it should meet $\partial D$ transversally. Under these hypotheses we construct a kernel $K_{M}^{h}(\zeta, z)$ so that for $f \in \mathcal{O}(M) \cap C(\bar{M})$ and $z \in M$ we have

$$
f(z)=\int_{\zeta \in \partial M} f(\zeta) K_{M}^{h}(\zeta, z) .
$$

This is the content of Theorem I.1.

The kernel $K_{M}^{h}$ is explicitly defined in terms of a function $\gamma(\gamma$ is assumed to satisfy (1) as in the Cauchy-Fantappiè formula) and in terms of functions $h_{i j}(\zeta, z)$, holomorphic in $(\zeta, z) \in U \times U$, so that

$$
h_{i}(\zeta)-h_{i}(z)=\sum_{j=1}^{m} h_{i j}(\zeta, z)\left(\zeta_{j}-z_{j}\right), \quad i=1, \ldots, m .
$$

(such functions always exist; see for example Harvey [2], p. 89).

Some of the interesting features of Theorem I.1 is on the one hand the explicit form of the kernel $K_{M}^{h}(\zeta, z)$ and on the other hand the fact that $M$ is allowed to have, finitely many, singular points.

The case $m=0$ of Theorem I.1 is the formula (2) and the case $m=1$ of it was obtained by Stout [15]. In fact Stout's paper was the starting point of this work. The kernel obtained by Stout coincides with ours in the case $m=1$; this is not immediate however and in §II we show that they are indeed the same.

The proof of Theorem I.1 (given in this paper) is an extension of Stout's proof from the case $m=1$ to the general case. A second proof of Theorem I.1 is contained in Hatziafratis [3].

In §III we develop a Weil type integral formula for analytic polyhedra on analytic varieties. The main result of this section is Theorem III.1 which generalizes the Weil integral formula for analytic polyhedra in $\mathbf{C}^{n}$ (see $[14,16])$. To obtain this result we combine results from $\S I$ together with some standard techniques contained for example in Range-Siu [12].

As we pointed out before the results in this paper are related to those of Palm [11] and Henkin and Leiterer [6]. The setting of Henkin and Leiterer [6] is more general than ours (we allow, however, finitely many singular points on $M$ ). On the other hand our results are more explicit than theirs. In fact we do not know the relation between our results and those of Henkin-Leiterer and Palm.

Acknowledgment. I would like to express my warmest thanks to Professor Alexander Nagel for very helpful discussions. 
I. We will use the standard notation of differential forms (see for example [13], Chapter 16). We will use also determinants whose entries are differential forms. For the properties of these determinants see for example [1], p. 8. As usual we will denote the Jacobian

$$
\operatorname{det}\left(\frac{\partial h_{j}}{\partial \zeta_{i}}(\zeta)\right)_{1 \leq i, j \leq m} \text { by } \frac{\partial\left(h_{1}, \ldots, h_{m}\right)}{\partial\left(\zeta_{1}, \ldots, \zeta_{m}\right)} \text {. }
$$

Also $\Lambda_{k \neq j_{1}, \ldots, j_{m}} d \zeta_{k}$ denotes the differential form

$$
d \zeta_{1} \wedge \cdots\left(j_{1}\right) \cdots\left(j_{m}\right) \cdots \wedge d \zeta_{n},
$$

i.e., $\left(j_{l}\right)$ means that $d \zeta_{j_{l}}$ is omitted.

The space of holomorphic functions on $X$ is denoted by $\mathcal{O}(X)$ and the space of continuous functions on $Y$ is denoted by $C(Y)$.

Description of the setting. Let $D$ be a bounded pseudoconvex domàin with smooth boundary and let $\gamma$ be as in the "introduction". Let $U$ be an open neighborhood of $\bar{D}$ and let $h=\left(h_{1}, \ldots, h_{m}\right): U \rightarrow \mathbf{C}^{m}$ be an $m$-tuple of holomorphic functions, $(m<n)$. Define

$$
Z(h)=:\left\{z \in U: h_{1}(z)=\cdots=h_{m}(z)=0\right\}
$$

and set

$$
M=: Z(h) \cap D \quad \text { and } \quad \partial M=: Z(h) \cap(\partial D) .
$$

Let $h_{i j}(\zeta, z)$ be holomorphic functions in $(\zeta, z) \in U \times U$ so that

$$
h_{i}(\zeta)-h_{i}(z)=\sum_{j=1}^{n} h_{i j}(\zeta, z)\left(\zeta_{j}-z_{j}\right), \quad i=1, \ldots, m .
$$

Consider the differential forms

$$
\begin{aligned}
& \alpha^{h}(\zeta, z)=: \operatorname{det}(\gamma_{j}, h_{1 j}, \ldots, h_{m j}^{4}, \overbrace{\bar{\partial}_{\zeta} \gamma_{j}, \ldots, \bar{\partial}_{\zeta} \gamma_{j}}^{n-m+1}) \\
& \beta^{h}(\zeta)=:(-1)^{m(m+1) / 2}|\nabla(h)(\zeta)|^{-2} \operatorname{det}\left(\mid \frac{\overline{\partial h_{1}}}{\partial \zeta_{j}}, \ldots, \frac{\overline{\partial h_{m}}}{\partial \zeta_{j}}, \frac{n-m}{d \zeta_{j}, \ldots, d \zeta_{j}}\right)
\end{aligned}
$$

(in each of the above determinants $j$ runs from $j=1$ to $j=n$ forming the 1st up to the $n$th row of it) where

$$
|\nabla(h)(\zeta)|^{2}=: \sum_{1 \leq j_{1}<\cdots<j_{m} \leq n}\left|\frac{\partial\left(h_{1}, \ldots, h_{m}\right)}{\partial\left(\zeta_{j_{1}}, \ldots, \zeta_{j_{m}}\right)}(\zeta)\right|^{2} .
$$

Now we introduce the kernel

$$
K_{M}^{h}(\zeta, z)=: c(n, m) \frac{\alpha^{h}(\zeta, z) \wedge \beta^{h}(\zeta)}{(\zeta-z, \gamma(\zeta, z))^{n-m}}
$$


where

$$
c(n, m)=:(-1)^{m(n+1)} \cdot(-1)^{n(n-1) / 2+1} \cdot \frac{1}{(n-m) !(2 \pi i)^{n-m}} .
$$

The main result of this section is the following:

THEOREM I1. If $|\nabla h| \neq 0$ on $\partial M$ and $Z(h)$ meets $\partial D$ transversally then for $f \in \mathcal{O}(M) \cap C(\bar{M})$ and $z \in M$ we have the integral formula:

$$
f(z)=\int_{\zeta \in \partial M} f(\zeta) K_{M}^{h}(\zeta, z) .
$$

Comments. (i) It follows from the assumptions made about the variety $Z(h)$ that $\partial M$ is a smooth $(2 n-2 m-1)$-dimensional manifold. Notice that we allow $Z(h)$ to have finitely many singular points in $D$.

(ii) Notice that if $m=0$ the integral formula is reduced to the Cauchy-Fantappiè formula.

(iii) Since we have fixed $\gamma$ we do not indicate the dependence of the forms $\alpha^{h}$ and $K_{M}^{h}$ on $\gamma$. These forms depend also on the factorizing functions $h_{i j}$ which we consider fixed too.

(iv) Notice also that if $\gamma_{j}(\zeta, z)$ are holomorphic functions of $z$ then the kernel $K_{M}^{h}(\zeta, z)$ is holomorphic in $z$ too.

The proof of Theorem I1 will be based on Theorem I2 which expresses an interesting "exactness" property of the kernels $K_{M}^{h}(\zeta, z)$. Roughly speaking, using Theorem I2 we will reduce the proof of Theorem I1 from the case with $\operatorname{codim} M=m$ to the case with $\operatorname{codim} M=m-1$, then to the case with $\operatorname{codim} M=m-2$ and so on until $\operatorname{codim} M=0$ in which case $M$ becomes $D$ and the integral formula of Theorem I1 is the Cauchy-Fantappiè formula.

In order to state Theorem 12 we need the following:

Let $g \in \mathcal{O}(U)$ and $g_{j} \in \mathcal{O}(U \times U)$ so that

$$
g(\zeta)-g(z)=\sum_{j=1}^{n} g_{j}(\zeta . z)\left(\zeta_{j}-z_{j}\right)
$$

Set $h^{*}=:\left(g, h_{1}, \ldots, h_{m}\right): U \rightarrow \mathbf{C}^{m+1}$ (now we assume that $m+1<n$ ) and $M^{*}=: Z\left(h^{*}\right) \cap D$.

Now we associate to $h^{*}, M^{*}$ (and the chosen factorizations of $\left.g, h_{1}, \ldots, h_{m}\right)$ the differential forms $\alpha^{h^{*}}, \beta^{h^{*}}$ and $K_{M^{*}}^{h^{*}}$ like before. In particular

$$
K_{M^{*}}^{h^{*}}=c(n, m+1) \frac{\alpha^{h^{*}} \wedge \beta^{h^{*}}}{(\zeta-z, \gamma)^{n-m-1}} \quad(\text { notice } m \text { is replaced by } m+1) \text {. }
$$


Now we state Theorem I2.

THEOREM I2. For a fixed $z \in M^{*}$ the following formula holds:

$$
d_{\zeta}\left[K_{M^{*}}^{h^{*}}(\zeta, z) \wedge \frac{d g(\zeta)}{g(\zeta)}\right]=2 \pi i K_{M}^{h}(\zeta, z)
$$

provided that the differential forms are restricted to $Z(h)-Z(g)$ locally at a point where $\left|\nabla h^{*}\right| \neq 0$.

Comments. (i) Notice that $\left|\nabla h^{*}(\zeta)\right| \neq 0$ implies $|\nabla h(\zeta)| \neq 0$. This is necessary for the definition of $K_{M^{*}}^{h^{*}}$ and $K_{M}^{h}$.

(ii) Since $\gamma(\zeta, z)$ is a smooth function of $\zeta \in \partial D$, if we fix $z$ in $D$ then there is a smooth extension of $\gamma(\zeta, z)$ for $\zeta$ in a neighborhood $W$ of $\partial D$ and it is with that extension that we will be working. We may also assume that $z \notin W$ and that $(\zeta-z, \gamma(\zeta, z)) \neq 0$ for $\zeta \in W$.

For the proof of Theorem I 2 we need the following lemmas.

LEMMA 1. We have

$$
\begin{aligned}
&\left(\sum_{j_{0}<\cdots<j_{m}}(-1)^{j_{0}+\cdots+J_{m}} \frac{\overline{\partial\left(g, h_{1}, \ldots, h_{m}\right)}}{\partial\left(\zeta_{j_{0}}, \zeta_{j_{1}}, \ldots, \zeta_{j_{m}}\right)} \bigwedge_{k \neq j_{0}, \ldots, j_{m}} d \zeta_{k}\right) \\
& \wedge\left(\sum_{l=1}^{n} \frac{\partial g}{\partial \zeta_{l}} d \zeta_{l}\right)=(-1)^{n+m} \sum_{j_{1}<\cdots<j_{m}}(-1)^{j_{1}+\cdots+j_{m}} \\
& \times\left[\sum_{l=1}^{n} \frac{\partial g}{\partial \zeta_{l}} \frac{\overline{\partial\left(g, h_{1}, \ldots, h_{m}\right)}}{\partial\left(\zeta_{l}, \zeta_{j_{1}}, \ldots, \zeta_{J_{m}}\right)}\right] \bigwedge_{k \neq j_{1}, \ldots, j_{m}} d \zeta_{k} .
\end{aligned}
$$

Proof. This is a straightforward computation. (Fix a multi-index $\left(j_{1}, \ldots, j_{m}\right)$ with $1 \leq j_{1}<\cdots<j_{m} \leq n$ and compute the coefficient of $\Lambda_{k \neq \lambda_{1}, \ldots, J_{m}} d \zeta_{k}$ in the left-hand side of (1).)

LEMMA 2. Consider the differential form

$$
\eta(\zeta)=: \sum_{j_{1}<\cdots<j_{m}}(-1)^{j_{1}+\cdots+j_{m}} a_{j_{1} \cdots j_{m}} \bigwedge_{k \neq j_{1}, \ldots, j_{m}} d \zeta_{k}
$$

where $a_{j_{1} \ldots j_{m}}$ are some smooth functions. Then $\eta(\zeta)$, restricted to $Z(h)$ locally at a point $\zeta^{0}$ with $\left(\partial\left(h_{1}, \ldots, h_{m}\right) / \partial\left(\zeta_{n-m+1}, \ldots, \zeta_{n}\right)\right)\left(\zeta^{0}\right) \neq 0$, can be expressed in the following way:

$$
\eta(\zeta)=A(\zeta) d \zeta_{1} \wedge \cdots \wedge d \zeta_{n-m}
$$


where

$$
A(\zeta)=: c^{*}\left(\frac{\partial\left(h_{1}, \ldots, h_{m}\right)}{\partial\left(\zeta_{n-m+1}, \ldots, \zeta_{n}\right)}\right)^{-1} \sum_{j_{1}<\cdots<j_{m}} a_{j_{1} \cdots j_{m}} \frac{\partial\left(h_{1}, \ldots, h_{m}\right)}{\partial\left(\zeta_{j_{1}}, \ldots, \zeta_{j_{m}}\right)}
$$

and

$$
c^{*}=:(-1)^{m(n-m)}(-1)^{m(m+1) / 2} .
$$

COROLlaRY 1. If $\zeta^{0}$ is as above then the differential form $\beta^{h}(\zeta)$, restricted to $Z(h)$ locally at $\zeta^{0}$, is given by the following formula:

$$
\beta^{h}(\zeta)=(n-m) ! \cdot c^{*}\left(\frac{\partial\left(h_{1}, \ldots, h_{m}\right)}{\partial\left(\zeta_{n-m+1}, \ldots, \zeta_{n}\right)}(\zeta)\right)^{-1} d \zeta_{1} \wedge \cdots \wedge d \zeta_{n-m} .
$$

Proof. It follows from the definition of $\beta^{h}(\zeta)$ that

$$
\beta^{h}(\zeta)=\frac{(n-m) !}{|\nabla h(\zeta)|^{2}} \sum_{j_{1}<\cdots<j_{m}}(-1)^{j_{1}+\cdots+j_{m}} \frac{\overline{\partial\left(h_{1}, \ldots, h_{m}\right)}}{\partial\left(\zeta_{j_{1}}, \ldots, \zeta_{j_{m}}\right)} \bigwedge_{k \neq j_{1}, \ldots, j_{m}} d \zeta_{k} .
$$

Thus Corollary 1 follows immediately from Lemma 2 applied with

$$
a_{j_{1} \cdots j_{m}}=: \frac{\overline{\partial\left(h_{1}, \ldots, h_{m}\right)}}{\partial\left(\zeta_{J_{1}}, \ldots, \zeta_{j_{m}}\right)} .
$$

Corollary 2. We have

$$
\beta^{h^{*}}(\zeta) \wedge d g(\zeta)=\frac{(-1)^{n+m}}{n-m} \beta^{h}(\zeta)
$$

when differential forms are restricted to $Z(h)$ locally at a point where $\left|\nabla h^{*}\right| \neq 0 .\left(\right.$ Recall $\left|\nabla h^{*}\right| \neq 0$ implies $|\nabla h| \neq 0$.)

Proof. Let $\zeta^{0}$ be as in Lemma 2. By Lemma 1 and the definition of $\beta^{h^{*}}(\zeta)$ we have

(1) $\beta^{h^{*}}(\zeta) \wedge d g(\zeta)=(-1)^{n+m} \frac{(n-m-1) !}{\left|\nabla h^{*}\right|^{2}}$

$$
\times \sum_{j_{1}<\cdots<j_{m}}(-1)^{j_{1}+\cdots+j_{m}} a_{j_{1} \cdots j_{m}} \bigwedge_{k \neq j_{1}, \ldots, j_{m}} d \zeta_{k}
$$

where we have set

$$
a_{h_{1} \ldots j_{m}}=: \sum_{l=1}^{n} \frac{\partial g}{\partial \zeta_{l}} \frac{\overline{\partial\left(g, h_{1}, \ldots, h_{m}\right)}}{\partial\left(\zeta_{l}, \zeta_{j_{1}}, \ldots, \zeta_{j_{m}}\right)}
$$


But it is easy to check that

$$
\sum_{j_{1}<\cdots<j_{m}} a_{j_{1} \cdots j_{m}} \frac{\partial\left(h_{1}, \ldots, h_{m}\right)}{\partial\left(\zeta_{j_{1}}, \ldots, \zeta_{j_{m}}\right)}=\left|\nabla h^{*}\right|^{2} .
$$

Also applying Lemma 2 with the $a_{j_{1} \cdots j_{m}}$ given by (2) using also (1) and (3) we obtain

(4) $\beta^{h^{*}}(\zeta) \wedge d g(\zeta)$

$$
=(n-m-1) !(-1)^{n+m} c^{*}\left(\frac{\partial\left(h_{1}, \ldots, h_{m}\right)}{\partial\left(\zeta_{n-m+1}, \ldots, \zeta_{n}\right)}\right)^{-1} \bigwedge_{k=1}^{n-m} d \zeta_{k} .
$$

Now (4) together with Corollary 1 completes the proof of Corollary 2.

For the proof of Lemma 2 we need the following elementary lemma.

Lemma 3. Let $A \in \mathbf{C}^{m \times m}$ be an $m \times m$ matrix, say,

$$
A=\left[\begin{array}{ccc}
\alpha_{11} & \cdots & \alpha_{1 m} \\
\vdots & & \vdots \\
\alpha_{m 1} & \cdots & \alpha_{m m}
\end{array}\right] \text { with } \operatorname{det}(A) \neq 0
$$

Let $B$ and $X \in \mathbf{C}^{m \times k}$ be $m \times k$ matrices, say,

$$
B=\left[\begin{array}{ccc}
b_{11} & \cdots & b_{1 k} \\
\vdots & & \vdots \\
b_{m 1} & \cdots & b_{m k}
\end{array}\right] \text { and } X=\left[\begin{array}{ccc}
x_{1}^{1} & \cdots & x_{1}^{k} \\
\vdots & & \vdots \\
x_{m}^{1} & \cdots & x_{m}^{k}
\end{array}\right] \text {. }
$$

Assume that

$$
\left.\begin{array}{l}
\alpha_{11} x_{1}^{j}+\cdots+\alpha_{1 m} x_{m}^{j}=b_{1 j} \\
\vdots \\
\alpha_{m 1} x_{1}^{j}+\cdots+\alpha_{m m} x_{m}^{j}=b_{m j}
\end{array}\right\} \quad j=1, \ldots, k
$$

i.e., we have $k$ systems of $m$ equations in $m$ unknowns each. Then

$$
\operatorname{det}\left[\begin{array}{ccc}
x_{1}^{1} & \cdots & x_{1}^{k} \\
\vdots & & \vdots \\
x_{k}^{1} & \cdots & x_{k}^{k}
\end{array}\right]=\frac{\operatorname{det}(C)}{\operatorname{det}(A)}
$$

where $C$ is the matrix obtained from $A$ by substituting the first $k$ columns of $A$ by the columns of $B$. 
Proof. Consider the $m \times m$ matrix $T$ :

$$
T=:\left[\begin{array}{cccccc}
x_{1}^{1} & \cdots & x_{1}^{k} & 0 & \cdots & 0 \\
\vdots & & \vdots & \vdots & & \vdots \\
x_{k}^{1} & \cdots & x_{k}^{k} & 0 & \cdots & 0 \\
x_{k+1}^{1} & \cdots & x_{k+1}^{k} & 1 & \cdots & 0 \\
\vdots & & \vdots & \vdots & & \vdots \\
x_{m}^{1} & \cdots & x_{m}^{k} & 0 & \cdots & 1
\end{array}\right] .
$$

Then using equations (1) it is easy to check that

$$
A \cdot T=C
$$

( $A \cdot T$ denotes the product of the matrices $A$ and $T$ ) and therefore

$$
\operatorname{det}(T)=\frac{\operatorname{det}(C)}{\operatorname{det}(A)}
$$

But

$$
\operatorname{det}(T)=\operatorname{det}\left[\begin{array}{ccc}
x_{1}^{1} & \cdots & x_{1}^{k} \\
\vdots & & \vdots \\
x_{k}^{1} & \cdots & x_{k}^{k}
\end{array}\right] .
$$

This completes the proof of the lemma.

Proof of Lemma 2. Throughout this proof we are working on $Z(h)$ locally at $\zeta^{0}$. Since $\left(\partial\left(h_{1}, \ldots, h_{m}\right) / \partial\left(\zeta_{n-m+1}, \ldots, \zeta_{n}\right)\right)\left(\zeta^{0}\right) \neq 0$ it follows from the implicit function theorem that there are holomorphic functions $\tilde{h}_{1}, \ldots, \tilde{h}_{m}$ of $\zeta^{(m)}=:\left(\zeta_{1}, \ldots, \zeta_{n-m}\right)$ so that the system of equations $\left\{h_{1}=\right.$ $\left.0, \ldots, h_{m}=0\right\}$ is equivalent to the system of the equations:

$$
\zeta_{n-m+J}=\tilde{h}_{j}\left(\zeta^{(m)}\right), \quad j=1, \ldots, m \quad\left(\text { locally at } \zeta^{0}\right) .
$$

In particular

$$
h_{j}\left(\zeta_{1}, \ldots, \zeta_{n-m}, \tilde{h}_{1}\left(\zeta^{(m)}\right), \ldots, \tilde{h}_{m}\left(\zeta^{(m)}\right)=0, \quad j=1, \ldots, m\right.
$$

which give the following equations:

(1) $\frac{\partial h_{j}}{\partial \zeta_{n-m+1}} \frac{\partial \tilde{h}_{1}}{\partial \zeta_{l}}+\cdots+\frac{\partial h_{j}}{\partial \zeta_{n}} \frac{\partial \tilde{h}_{m}}{\partial \zeta_{l}}=-\frac{\partial h_{j}}{\partial \zeta_{l}}$

$$
j=1, \ldots, m, l=1, \ldots, n-m \text {. }
$$


Now fix a multi-index $\left(j_{1}, \ldots, j_{m}\right)$ with $1 \leq j_{1}<\cdots<j_{m} \leq n$. Let us assume that

$$
1 \leq j_{1}<\cdots<j_{k} \leq n-m<n-m+1 \leq j_{k+1}<\cdots<j_{m} \leq n
$$

( $k$ can be any one of the numbers $1,2, \ldots, m$ ).

Let us consider the differential form

$$
\begin{aligned}
\theta= & d \zeta_{1} \wedge \cdots\left(j_{1}\right) \cdots\left(j_{k}\right) \cdots \wedge d \zeta_{n-m} \wedge d \zeta_{n-m+1} \\
& \wedge \cdots\left(j_{k+1}\right) \cdots\left(j_{m}\right) \cdots \wedge d \zeta_{n} .
\end{aligned}
$$

Recall that $\left(j_{l}\right)$ in $\theta$ means that $d \zeta_{j_{l}}$ is omitted. Recall also that differential forms are considered restricted to $Z(h)$ locally at $\xi^{0}$. Thus $\theta$ is a "multiple" of $d \zeta_{1} \wedge \cdots \wedge d \zeta_{n-m}$. In fact

$$
\boldsymbol{\theta}=\sigma_{1} \cdot \frac{\partial\left(\tilde{h}_{1}, \ldots, \hat{j}_{k+1}, \ldots, \hat{j}_{m}, \ldots, \tilde{h}_{m}\right)}{\partial\left(\zeta_{j_{1}}, \ldots, \zeta_{j_{k}}\right)} \bigwedge_{k=1}^{n-m} d \zeta_{k}
$$

where $\sigma_{1}$ is the sign so that

$$
\begin{gathered}
d \zeta_{1} \wedge \cdots\left(j_{1}\right) \cdots\left(j_{k}\right) \cdots \wedge d \zeta_{n-m} \wedge d \zeta_{j_{1}} \wedge \cdots \wedge d \zeta_{J_{k}} \\
=\sigma_{1} d \zeta_{1} \wedge \cdots \wedge d \zeta_{n-m}
\end{gathered}
$$

(in the Jacobian of (2), “ $\hat{j}_{k+1}$ " means that $\tilde{h}_{j_{k+1}-(n-m)}$ is omitted and similarly for $\hat{j}_{k+2}, \ldots, \hat{j}_{m}$ ).

It is easy to compute $\sigma_{1}$ :

$$
\sigma_{1}=(-1)^{(m-n) k}(-1)^{J_{1}+\cdots+j_{k}}(-1)^{k(k-1) / 2} .
$$

Next applying Lemma 3 with the systems of equations (1) (with $\partial \tilde{h}_{i} / \partial \zeta_{j}$ in the place of the $x$ 's) we obtain

$$
\begin{aligned}
& \frac{\partial\left(\tilde{h}_{1}, \ldots, \hat{j}_{k+1}, \ldots, \hat{j}_{m}, \ldots, \tilde{h}_{m}\right)}{\partial\left(\zeta_{J_{1}}, \ldots, \zeta_{j_{k}}\right)} \\
& \quad=(-1)^{k} \cdot \sigma_{2} \cdot \frac{\partial\left(h_{1}, \ldots, h_{m}\right)}{\partial\left(\zeta_{j_{1}}, \ldots, \zeta_{j_{m}}\right)}\left(\frac{\partial\left(h_{1}, \ldots, h_{m}\right)}{\partial\left(\zeta_{n-m+1}, \ldots, \zeta_{n}\right)}\right)^{-1}
\end{aligned}
$$

where $\sigma_{2}$ is the sign of the following permutation:

$$
\sigma_{2}=\operatorname{sign}\left[\begin{array}{c}
n-m+1, \ldots, \hat{j}_{k+1}, \ldots, \hat{j}_{m}, \ldots, n, j_{k+1}, \ldots, j_{m} \\
n-m+1, \ldots, n
\end{array}\right] .
$$

It is easy to compute $\sigma_{2}$ :

$$
\sigma_{2}=(-1)^{n(m-k)} \cdot(-1)^{j_{k+1}+\cdots+j_{m}} \cdot(-1)^{(m-k)(m-k-1) / 2} .
$$


Now from (2), (3), (4) and (5) we obtain

$$
\begin{aligned}
(-1)^{J_{1}+\cdots+J_{m}} \bigwedge_{l \neq j_{1}, \ldots, j_{m}} d \zeta_{l} \\
\quad=c^{*} \frac{\partial\left(h_{1}, \ldots, h_{m}\right)}{\partial\left(\zeta_{j_{1}}, \ldots, \zeta_{j_{m}}\right)}\left(\frac{\partial\left(h_{1}, \ldots, h_{m}\right)}{\partial\left(\zeta_{n-m+1}, \ldots, \zeta_{n}\right)}\right)^{-1} \bigwedge_{k=1}^{n-m} d \zeta_{k}
\end{aligned}
$$

which implies the formula of Lemma 2 and completes the proof.

LEMMA 4. If $\phi:(\partial D) \times D \rightarrow \mathbf{C}-\{0\}$ is a smooth function then

$$
\operatorname{det}(\phi \gamma_{j}, h_{1 j}, \ldots, h_{m j}, \overbrace{\bar{\partial}_{\zeta}\left(\phi \gamma_{j}\right), \ldots, \bar{\partial}_{\zeta}\left(\phi \gamma_{j}\right)}^{n-m-1})=\phi^{n-m} \cdot \alpha^{h}(\zeta, z) .
$$

In particular,

$$
(\zeta-z, \gamma)^{m-n} \cdot \alpha^{h}(\zeta, z)=\operatorname{det}(\tilde{\gamma}_{j}, h_{1 j}, \ldots, h_{m j}, \overbrace{\bar{\partial}_{\zeta} \tilde{\gamma}_{j}, \ldots, \bar{\partial}_{\zeta} \tilde{\gamma}_{j}}^{n-m-1})
$$

where $\tilde{\gamma}_{j}=(\zeta-z, \gamma)^{-1} \cdot \gamma_{j}, j=1, \ldots, n$.

Proof. This follows from properties of determinants with entries differential forms (see [1], p. 8).

We now turn to the proof of Theorem I2.

Proof of Theorem I2. Throughout this proof differential forms are restricted (in $\zeta$ ) to $Z(h)-Z(g)$ locally at a point where $\left|\nabla h^{*}\right| \neq 0$ and $z$ is a fixed point on $M^{*}$. In view of Lemma 4 we may and do assume that

$$
(\zeta-z, \gamma(\zeta, z))=1 \quad \text { for }(\zeta, z) \in(\partial D) \times D .
$$

Next we claim that

$$
\bar{\partial}_{\zeta}\left[\alpha^{h^{*}}(\zeta, z)\right]=(-1)^{m+1} \cdot g(\zeta) \cdot \alpha^{h}(\zeta, z) .
$$

Now we prove (2). We have

$$
\boldsymbol{\alpha}^{h^{*}}(\zeta, z)=\operatorname{det}\left[\begin{array}{llll}
\gamma_{1} & g_{1} & h_{11} \cdots h_{m 1} & \frac{n-m-2}{\bar{\partial} \gamma_{1} \cdots \bar{\partial} \gamma_{1}} \\
\gamma_{j} & g_{j} & h_{1 j} \cdots h_{m j} & \bar{\partial} \gamma_{j} \cdots \bar{\partial} \gamma_{j}
\end{array}\right]
$$

(in the above determinant $j$ runs from $j=2$ to $j=n$ forming the 2 nd up to the $n$th row of it).

We may assume $\zeta_{1} \neq z_{1}$. Then, by (1), we have

$$
\left(\zeta_{1}-z_{1}\right) \alpha^{h^{*}}(\zeta, z)=\operatorname{det}\left[\begin{array}{cccc}
1 & g(\zeta) & 0 \cdots 0 & \overbrace{0 \cdots 0}^{n-m-2} \\
\gamma_{j} & g_{J} & h_{1 j} \cdots h_{m j} & \bar{\partial} \gamma_{j} \cdots \bar{\partial} \gamma_{j}
\end{array}\right]
$$


$\left(\zeta_{1}-z_{1}\right.$ multiplied the first row and we added to the first row of the determinant the $j$ th-rows multiplied by $\left.\left(\zeta_{j}-z_{j}\right), j=2, \ldots, n\right)$ and therefore

$$
\begin{aligned}
& \left(\zeta_{1}-z_{1}\right) \bar{\partial}_{\zeta}\left(\alpha^{h^{*}}(\zeta, z)\right) \\
& =\operatorname{det}\left[\begin{array}{cccc}
0 & g(\zeta) & 0 \cdots 0 & \overbrace{0 \cdots 0}^{n-m-2} \\
\bar{\partial} \gamma_{j} & g_{j} & h_{1 j} \cdots h_{m j} & \bar{\partial} \gamma_{j} \cdots \bar{\partial} \gamma_{j}
\end{array}\right] \\
& =(-1)^{m+1} \operatorname{det}\left[\begin{array}{ccc}
g(\zeta) & 0 \cdots 0 & \overbrace{0 \cdots 0}^{n-m-1} \\
g_{J} & h_{1 j} \cdots h_{m j} & \bar{\partial} \gamma_{j} \cdots \bar{\partial} \gamma_{j}
\end{array}\right] \\
& =(-1)^{m+1} g(\zeta) \operatorname{det}[h_{1 j}, \ldots, h_{m j}, \overbrace{\overline{\bar{\partial}} \gamma_{j}, \ldots, \bar{\partial} \gamma_{j}}^{n-m-1}]
\end{aligned}
$$

(the last determinant in (3) is $(n-1) \times(n-1)$ and $j$ runs from $j=2$ to $j=n$ forming the $(n-1)$ rows of it). On the other hand

$$
\begin{aligned}
& \text { (4) } \quad\left(\zeta_{1}-z_{1}\right) \alpha^{h}(\zeta, z)=\left(\zeta_{1}-z_{1}\right) \operatorname{det}\left[\begin{array}{lll}
\gamma_{1} & h_{11} \cdots h_{m 1} & \frac{n-m-1}{\bar{\partial} \gamma_{1} \cdots \bar{\partial} \gamma_{1}} \\
\gamma_{j} & h_{1 j} \cdots h_{m J} & \bar{\partial} \gamma_{j} \cdots \bar{\partial} \gamma_{j}
\end{array}\right] \\
& =\operatorname{det}\left[\begin{array}{ccc} 
& 0 \cdots 0 & \frac{n-m-1}{0 \cdots 0} \\
\gamma_{j} & h_{1 j} \cdots h_{m j} & \bar{\partial} \gamma_{j} \cdots \bar{\partial} \gamma_{j}
\end{array}\right] \\
& =\operatorname{det}[h_{1 j}, \ldots, h_{m j}, \overbrace{\overline{\bar{\partial}} \gamma_{j}, \ldots, \bar{\partial} \gamma_{j}}^{n-m-1}] \text {. }
\end{aligned}
$$

But (3) and (4) imply (2). This proves (2). Now the proof of Theorem I2 follows from (2) and Corollary 2 (recall that differential forms are restricted to $Z(h)-Z(g))$.

This concludes the proof of Theorem I2.

Finally we turn to the proof of Theorem I1. As we pointed out earlier this proof is based on ideas of Stout. See also [15] which contains the case $m=1$

Proof of Theorem I1. First we make a few reductions. Since we assume that $D$ is pseudoconvex, in view of Cartan's extension theorem and an approximation argument (recall that a pseudoconvex domain is the union 
of an increasing sequence of strictly pseudoconvex domains) we may and do assume that $f \in \mathcal{O}(\bar{D})$, i.e., $f$ is holomorphic in a neighborhood of $\bar{D}$.

Also Theorem I1 is true for $m=0$ (since for $m=0$ it is the Cauchy-Fantappiè formula) and therefore we can prove it by assuming that it is true for the case when the variety is defined by $m$ holomorphic functions and proving it when the variety is defined by $m+1$ holomorphic functions, i.e., we will assume it for $M$ and we will prove it for $M^{*}$. Thus our assumptions are that $\left|\nabla h^{*}\right| \neq 0$ on $\partial M^{*}$ and that $Z\left(h^{*}\right)$ meets $\partial D$ transversally.

Moreover in view of Sard's theorem on the set of critical points (using a deformation argument) and by the implicit function theorem we may and do assume that:

(i) $|\nabla h| \neq 0$ on $\partial M$

(ii) $Z(h)$ meets $\partial D$ transversally.

Notice also that $Z\left(h^{*}\right)$ meets $\partial M$ transversally. It follows from these assumptions that $\partial M$ and $\partial M^{*}$ are smooth manifolds of dimensions $(2 n-2 m-1)$ and $(2 n-2 m-3)$ respectively. It also follows that $\{\zeta \in$ วM: $g(\zeta)=\tau\}$ is a smooth manifold (for $\tau \in \mathbf{C},|\tau|<\varepsilon$ and $\varepsilon$ a small positive number) diffeomorphic to $\partial M^{*}$.

Since we assume Theorem I1 for $M$ we have

$$
f(z)=\int_{\zeta \in \partial M} f(\zeta) K_{M}^{h}(\zeta, z) .
$$

Next write (1) as follows

$$
f(z)=\left(\int_{\zeta \in(\partial M)_{\varepsilon}}+\int_{\zeta \in(\partial M) \backslash(\partial M)_{\varepsilon}}\right)\left(f(\zeta) K_{M}^{h}(\zeta, z)\right)
$$

where $(\partial M)_{\varepsilon}=:\{\zeta \in \partial M:|g(\zeta)|>\varepsilon\}(\varepsilon>0$, small). But

$$
\lim _{\varepsilon \rightarrow 0} \int_{\zeta \in(\partial M)-(\partial M)_{\varepsilon}} f(\zeta) K_{M}^{h}(\zeta, z)=0
$$

and (2) becomes

$$
f(z)=\lim _{\varepsilon \rightarrow 0} \int_{\zeta \in(\partial M)_{\varepsilon}} f(\zeta) K_{M}^{h}(\zeta, z) .
$$

Next by Theorem $\mathrm{I} 2$ and the fact that $f$ is holomorphic we obtain

$$
d_{\zeta}\left[f(\zeta) K_{M^{*}}^{h^{*}}(\zeta, z) \wedge \frac{d g(\zeta)}{g(\zeta)}\right]=2 \pi i f(\zeta) K_{M}^{h}(\zeta, z)
$$


on $Z(h)-Z(g)$ and therefore

(4)

$$
\begin{aligned}
& \int_{\zeta \in(\partial M)_{\varepsilon}} f(\zeta) K_{M}^{h}(\zeta, z)=\frac{1}{2 \pi i} \int_{\zeta \in(\partial M)_{\varepsilon}} d_{\zeta}\left[f(\zeta) K_{M^{*}}^{h^{*}}(\zeta, z) \wedge \frac{d g(\zeta)}{g(\zeta)}\right] \\
& =\frac{1}{2 \pi i} \int_{\zeta \in \partial(\partial M)_{\varepsilon}} f(\zeta) K_{M^{*}}^{h^{*}}(\zeta, z) \wedge \frac{d g(\zeta)}{g(\zeta)} \quad \text { (by Stokes' theorem) } \\
& \quad=\frac{1}{2 \pi i} \int_{|\tau|=\varepsilon}\left(\int_{\{\zeta \in \partial M: g(\zeta)=\tau\}} f(\zeta) K_{M^{*}}^{h^{*}}(\zeta, z)\right) \frac{d \tau}{\tau} .
\end{aligned}
$$

Letting $\varepsilon \rightarrow 0$ in (4) we see that

$$
\begin{aligned}
\lim _{\varepsilon \rightarrow 0} \int_{\zeta \in(\partial M)_{\varepsilon}} f(\zeta) K_{M}^{h}(\zeta, z) & =\int_{\{\zeta \in \partial M: g(\zeta)=0\}} f(\zeta) K_{M^{*}}^{h^{*}}(\zeta, z) \\
& =\int_{\zeta \in \partial M^{*}} f(\zeta) K_{M^{*}}^{h^{*}}(\zeta, z) .
\end{aligned}
$$

Now (3) and (5) imply that

$$
f(z)=\int_{\zeta \in \partial M^{*}} f(\zeta) K_{M^{*}}^{h^{*}}(\zeta, z) .
$$

Thus the integral formula holds for $M^{*}$ and the proof of Theorem I1 is complete.

II. In this section we will show that the kernel $K_{M}^{h}(\zeta, z)$ of Theorem I.1 with $m=1$ coincides with the kernel constructed in Stout [15]. Here $m=1$, i.e., the variety $M$ is defined by one holomorphic function. Let $D$, $U$ and $\gamma$ be as in Theorem I.1 and let $h \in \mathcal{O}(U)$ and $h_{j} \in \mathcal{O}(U \times U)$ so that

$$
h(\zeta)-h(z)=\sum_{j=1}^{n} h_{j}(\zeta, z)\left(\zeta_{j}-z_{j}\right) .
$$

Let $M=\{z \in D: h(z)=0\}$ and define

$$
\begin{aligned}
\alpha(\zeta, z)= & : \sum_{1 \leq i<j \leq n}(-1)^{i+\jmath+1}\left(h_{i} \gamma_{j}-h_{j} \gamma_{i}\right) \bar{\partial} \gamma_{1} \wedge \cdots(i) \cdots(j) \cdots \wedge \bar{\partial} \gamma_{n} \\
\beta(\zeta)= & : \frac{1}{|\nabla h(\zeta)|^{2}} \sum_{j=1}^{n}(-1)^{j} \frac{\overline{\partial h}}{\partial \zeta_{j}} d \zeta_{1} \wedge \cdots(j) \cdots \wedge d \zeta_{n} \\
& \left(|\nabla h(\zeta)|^{2}=: \sum_{j=1}^{n}\left|\frac{\partial h}{\partial \zeta_{j}}\right|^{2}\right)
\end{aligned}
$$


and

$$
K(\zeta, z)=:(-1)^{(n-1)(n-2) / 2+1} \frac{(n-2) !}{(2 \pi i)^{n-1}} \frac{\alpha(\zeta, z) \wedge \beta(\zeta)}{(\zeta-z, \gamma(\zeta, z))^{n-1}} .
$$

$K(\zeta, z)$ is our kernel in the case $m=1$. Stout defined the kernel in the following way:

$$
\text { (constant) }) \frac{\sum_{j=1}^{n}(-1)^{(n-1)(j-1)} h_{,} \alpha^{(\jmath)} \wedge \beta(\zeta)}{(\zeta-z, \gamma(\zeta, z))^{n-1}}
$$

where $\alpha^{(/)}$are the following differential forms:

$$
\alpha^{(1)}=: \sum_{k=2}^{n}(-1)^{k} \gamma_{k} \bar{\partial} \gamma_{2} \wedge \cdots(k) \cdots \wedge \bar{\partial} \gamma_{n}
$$

and the remaining $\alpha^{(j)}$ are defined by cyclic permutation of the $\gamma_{\text {, }}$ 's. Let us look in the following figure:

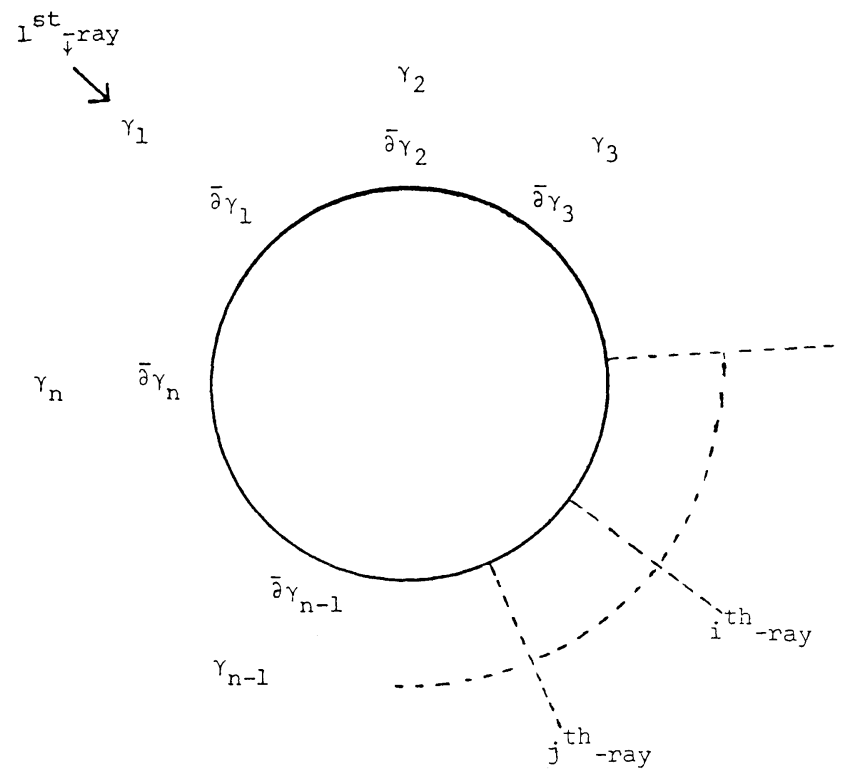

To define $\alpha^{(1)}$ we eliminated the first ray and we "expanded" the remaining in a way that is clear from the definition of $\alpha^{(1)}$. Thus to obtain $\alpha^{(2)}$ we eliminate the second ray and so on; for example

$$
\begin{aligned}
\alpha^{(2)}= & \gamma_{3} \bar{\partial} \gamma_{4} \wedge \cdots \wedge \bar{\partial} \gamma_{n} \wedge \bar{\partial} \gamma_{1}-\gamma_{4} \bar{\partial} \gamma_{3} \wedge \bar{\partial} \gamma_{5} \wedge \cdots \wedge \bar{\partial} \gamma_{n} \wedge \bar{\partial} \gamma_{1} \\
& +\cdots+(-1)^{n-1} \gamma_{n} \bar{\partial} \gamma_{3} \wedge \cdots \wedge \bar{\partial} \gamma_{n-1} \wedge \bar{\partial} \gamma_{1} \\
& +(-1)^{n} \gamma_{1} \bar{\partial} \gamma_{3} \wedge \cdots \wedge \bar{\partial} \gamma_{n}
\end{aligned}
$$

(see also Stout [15]). 
Thus in order to show that $K(\zeta, z)$ coincides with the Stout's kernel we have to show that

$$
\sum_{j=1}^{n}(-1)^{(n-1)(j-1)} h_{j} \alpha^{(j)}=\alpha .
$$

Indeed $\sum_{j=1}^{n}(-1)^{(n-1)(j-1)} h_{j} \alpha^{(j)}$ is a combination of the forms $\bar{\partial} \gamma_{1}$ $\wedge \cdots(i) \cdots(j) \cdots \wedge \bar{\partial} \gamma_{n}(1 \leq i<j \leq n)$. The form $\theta_{(i, j)}=: \bar{\partial} \gamma_{1}$ $\wedge \cdots(i) \cdots(j) \cdots \wedge \bar{\partial} \gamma_{n}$ comes from the forms $\alpha^{(t)}$ and $\alpha^{(j)}$ :

$\alpha^{(i)}$ is defined by eliminating the $i$ th-ray so we get the form $\theta_{(i, j)}$ at the $j$ th-ray stage of the expansion of $\alpha^{(i)}$ as $(-1)^{j-i+1} \gamma_{j} \bar{\partial} \gamma_{i+1} \wedge \cdots \wedge \bar{\partial} \gamma_{j-1} \wedge \bar{\partial} \gamma_{j+1} \wedge \cdots \wedge \bar{\partial} \gamma_{n} \wedge \bar{\partial} \gamma_{1} \wedge \cdots \wedge \bar{\partial} \gamma_{i-1}$

$$
=(-1)^{j-i+1}(-1)^{(i-1)(n-i-1)} \gamma_{j} \theta_{(i, j)} .
$$

$\boldsymbol{\alpha}^{(j)}$ is defined by eliminating the $j$ th-ray so we get the form $\theta(i, j)$ at the $i$ th-ray stage of the expansion of $\alpha^{(j)}$, as

$$
\begin{aligned}
(-1)^{n-j+i-1} \gamma_{i} \bar{\partial} \gamma_{j+1} & \wedge \cdots \wedge \bar{\partial} \gamma_{n} \wedge \bar{\partial} \gamma_{1} \wedge \cdots \wedge \bar{\partial} \gamma_{i-1} \wedge \bar{\partial} \gamma_{i+1} \wedge \cdots \wedge \bar{\partial} \gamma_{j-1} \\
& =(-1)^{n-j+i-1}(-1)^{(n-j)(j-2)} \gamma_{i} \theta_{(i, j)}
\end{aligned}
$$

Therefore the coefficient of $\theta(i, j)$ in $\sum_{j=1}^{n}(-1)^{(n-1)(j-1)} h_{j} \alpha^{(j)}$ is

$$
\begin{gathered}
(-1)^{(n-1)(i-1)}(-1)^{j-1+1}(-1)^{(i-1)(n-i-1)} h_{i} \gamma_{j} \\
+(-1)^{(n-1)(j-1)}(-1)^{(n-j+i-1)}(-1)^{(n-j)(j-2)} h_{j} \gamma_{i} \\
=(-1)^{i+j-1}\left(h_{i} \gamma_{j}-h_{j} \gamma_{i}\right) .
\end{gathered}
$$

This proves $(*)$.

III. In this section we develop a Weil type integral formula for analytic polyhedra on analytic varieties. We will do this by combining results and methods from $\$ I$ and from Range-Siu [12]. The main result of this section is Theorem III.1 which generalizes the Weil integral formula for analytic polyhedra in $\mathbf{C}^{n}$. (See Sommer [14]). We start by describing the setting. Let $h_{1}, \ldots, h_{m}, g_{1}, \ldots, g_{N}$ be holomorphic function on $\mathbf{C}^{n}$ with $m<n$ and $N \geq n-m$. Let

$$
V=:\left\{z \in \mathbf{C}^{n}: h_{1}(z)=\cdots=h_{m}(z)=0\right\}
$$

and

$$
P=\left\{z \in V:\left|g_{1}(z)\right|<1, \ldots,\left|g_{N}(z)\right|<1\right\}
$$


We denote by $\bar{P}$ the closure of $P$. Also

$$
\partial P=\left\{\zeta \in \bar{P}:\left|g_{j}(\zeta)\right|=1 \text { for some } j: 1 \leq j \leq N\right\}=: \bigcup_{j=1}^{N} \tau_{J} .
$$

More generally, for $J=\left\{j_{1}<\cdots<j_{k}\right\} \subset\{1, \ldots, N\}$ define

$$
\tau_{J}=:\left\{\zeta \in \bar{P}:\left|g_{j_{1}}(\zeta)\right|=\cdots=\left|g_{j_{k}}(\zeta)\right|=1\right\} .
$$

Now we make the following assumptions:

$\left(^{*}\right)|\nabla(h)| \neq 0$ on $\partial P\left(h=:\left(h_{1}, \ldots, h_{m}\right)\right.$ and $|\nabla h|$ is as in $\left.\S \mathrm{I}\right)$.

$\left.{ }^{* *}\right) \tau_{J}$ is assumed to be piecewise $C^{1}$-manifold for all $J$.

Next let us consider holomorphic functions $h_{i j}(\zeta, z)$ and $g_{i j}(\zeta, z)$ so that

$$
h_{i}(\zeta)-h_{i}(z)=\sum_{j=1}^{n} h_{i j}(\zeta, z)\left(\zeta_{j}-z_{j}\right), \quad 1 \leq i \leq m
$$

and

$$
g_{i}(\zeta)-g_{i}(z)=\sum_{j=1}^{n} g_{i j}(\zeta, z)\left(\zeta_{j}-z_{j}\right), \quad 1 \leq i \leq N
$$

Also let $H_{i}, G_{i}$ be the following columns: $H_{i}={ }^{t}\left(h_{i 1}, \ldots, h_{i n}\right), 1 \leq i \leq m$, $G_{i}={ }^{t}\left(g_{i 1}, \ldots, g_{\text {in }}\right), 1 \leq i \leq N$, and let $\beta^{h}(\zeta)$ be the differential form associated to $h$ as in $\S$ I.

With this notation and under the assumptions $\left({ }^{*}\right)$ and $\left({ }^{* *}\right)$ we will prove the following theorem.

TheOREM III.1. For $f \in \mathcal{O}(\bar{P})$, i.e., $f$ holomorphic in a neighborhood (in $V$ ) of $\bar{P}$ and $z \in P$ we have:

$$
f(z)=\tilde{c}(n, m) \sum_{1 \leq j_{1}<\cdots<j_{n-m} \leq N} \int_{\zeta \in \tau_{j_{1} \cdots j_{n-m}}} f(\zeta) W_{(\zeta, z)}^{\left(j_{1} \cdots j_{n-m}\right)} \beta^{h}(\zeta)
$$

where

$$
W_{(\zeta, z)}^{\left(j_{1}, \ldots, j_{n-m}\right)}=\frac{\operatorname{det}\left(H_{1}, \ldots, H_{m}, G_{j_{1}}, \ldots, G_{j_{n-m}}\right)}{\prod_{k=1}^{n-m}\left[g_{j_{k}}(\zeta)-g_{j_{k}}(z)\right]}
$$

and

$$
\tilde{c}(n, m)=(-1)^{m} \cdot(-1)^{(n+m-1)(n+m) / 2} \frac{1}{(n-m) !(2 \pi i)^{n-m}} .
$$


Before we prove the above theorem we introduce some further notation and give two lemmas. Consider the simplex

$$
\Delta=\left\{\lambda=\left(\lambda_{0}, \ldots, \lambda_{N}\right) \in \mathbf{R}^{N+1}: \lambda_{j} \geq 0 \text { and } \sum_{j=0}^{N} \lambda_{j}=1\right\} .
$$

For $J=\left\{j_{1}<\cdots<j_{k}\right\} \subset\{0,1, \ldots, N\}$ define the $J$-face of $\Delta$ by

$$
\Delta_{J}=\left\{\lambda \in \Delta: \sum_{l=1}^{k} \lambda_{j_{l}}=1\right\} \text {. }
$$

Lemma 1. Consider the chain $c=: \Sigma_{J}(-1)^{|J|} \tau_{J} \times \Delta_{0 J}$ where $\Sigma_{J}$ indicates that the summation is extended over all ordered multi-indices $J \subset$ $\{1, \ldots, N\}$. Then

$$
\partial c=\sum_{J} \tau_{J} \times \Delta_{J}-(\partial P) \times \Delta_{0}
$$

Proof. See Range-Siu [12], p. 329.

Next for $j \in\{1, \ldots, N\}$ define

$$
\gamma_{(\zeta, z)}^{J}=\left(\gamma_{1}^{J}, \ldots, \gamma_{n}^{J}\right) \quad \text { where } \gamma_{l}^{j}=\frac{g_{j l}}{g_{j}(\zeta)-g_{j}(z)}, \quad l=1, \ldots, n \text {. }
$$

Notice that $\gamma_{(\zeta, z)}^{j}$ is well-defined if $\left|g_{J}(\zeta)\right|=1$ and $\left|g_{J}(z)\right|<1$, i.e., if $z \in P$ and $\zeta \in \tau_{j}$. Also notice that

$$
\sum_{l=1}^{n} \gamma_{l}^{j}(\zeta, z)\left(\zeta_{l}-z_{l}\right)=1 \quad \text { for } j=1, \ldots, N \text {. }
$$

Also define

$$
\gamma_{(\zeta, z)}^{0}=\left(\gamma_{1}^{0}, \ldots, \gamma_{n}^{0}\right) \quad \text { where } \gamma_{l}^{0}=\frac{\bar{\zeta}_{l}-\bar{z}_{l}}{|\zeta-z|^{2}}, \quad l=1, \ldots, n .
$$

Now for $\lambda=\left(\lambda_{0}, \ldots, \lambda_{N}\right) \in \Delta$ let

$$
\gamma_{l}(\zeta, z, \lambda)=: \sum_{j=0}^{N} \lambda_{j} \gamma_{l}^{J}(\zeta, z), \quad l=1, \ldots, n .
$$

Notice that for $(\zeta, \lambda) \in \tau_{J} \times \Delta_{0 J}, \gamma(\zeta, z, \lambda)=:\left(\gamma_{1}, \ldots, \gamma_{n}\right)$ is well-defined and also that

$$
\sum_{l=1}^{n} \gamma_{l}(\zeta, z, \lambda)\left(\zeta_{l}-z_{l}\right)=1 \quad \text { for }(\zeta, \lambda) \in \tau_{J} \times \Delta_{0 J}
$$


We introduce the differential form

$$
K(\zeta, z, \lambda)=: \sum_{I}(-1)^{|I|} A^{I} \bigwedge_{l \notin I} \partial_{\zeta, \lambda}^{\prime} \gamma_{l} \wedge \beta^{h}(\zeta)
$$

where $\Sigma_{I}$ indicates that the summation is extended over ordered multi-indices $I=\left\{i_{0}<\cdots<i_{m}\right\} \subset\{1, \ldots, n\},|I|=i_{0}+\cdots+i_{m}, \partial_{\zeta, \lambda}^{\prime}=: \bar{\partial}_{\zeta}$ $+d_{\lambda}$ and $A^{I}$ is the following determinant:

$$
A^{I}=\left|\begin{array}{ccc}
h_{1 i_{0}} & \cdots & h_{1 i_{m}} \\
\vdots & & \vdots \\
h_{m i_{0}} & \cdots & h_{m i_{m}} \\
\gamma_{i_{0}} & \cdots & \gamma_{i_{m}}
\end{array}\right| .
$$

The differential form $K(\zeta, z, \lambda)$ can also be written in the following way:

$$
K(\zeta, z, \lambda)=\frac{1}{(n-m-1) !} \alpha(\zeta, z, \lambda) \wedge \beta^{h}(\zeta)
$$

where

$$
\alpha(\zeta, z, \lambda)=: \operatorname{det}(h_{1 j}, \ldots, h_{m j}, \gamma_{j}, \quad \overbrace{\partial_{\zeta, \lambda}^{\prime} \gamma_{j}, \ldots, \partial_{\zeta, \lambda}^{\prime} \gamma_{j}}^{n-m-1})
$$

(in the above determinant $j$ runs from $j=1$ to $j=n$ forming the 1 st up to the $n$th row of it).

LEMMA 2. We have

$$
d_{\zeta, \lambda}[K(\zeta, z, \lambda)]=0
$$

provided that differential forms are restricted in $(\zeta, \lambda)$ to the chain $c$ of Lemma 1 and $z$ is a fixed point on $P$.

Proof. Notice that

(2) $\quad\left(\zeta_{1}-z_{1}\right) \alpha(\zeta, z, \lambda)=\operatorname{det}\left[\begin{array}{ccccc}0 & \cdots & \cdots & 1 & \overbrace{0 \cdots 0}^{n-m-1} \\ h_{1 j} \cdots & h_{m j} & \gamma_{j} & \partial_{\zeta, \lambda}^{\prime} \gamma_{j} \cdots \partial_{\zeta, \lambda}^{\prime} \gamma_{j}\end{array}\right]$.

In the above determinant $j$ runs from $j=2$ to $j=n$ forming the 2 nd up to the $n$th row of it; we obtained (2) in the following way: $\left(\zeta_{1}-z_{1}\right)$ multiplied the first row of the determinant $\alpha(\zeta, z, \lambda)$ and then we added to this first row the remaining $j$ th-rows multiplied by $\zeta_{j}-z_{j}$; we also used (1) and the fact that $\zeta, z \in V$.

Now using (2) it is easy to show that

$$
\partial_{\zeta, \lambda}^{\prime}[\alpha(\zeta, z, \lambda)]=0
$$


On the other hand, by Corollary 1 of $§ I$ we have

$$
d \beta^{h}(\zeta)=0
$$

Now (3) and (4) complete the proof of Lemma 2.

Proof of Theorem III.1. Apply Stokes' theorem to the chain $c$ of Lemma 1 and to the differential form $f(\zeta) K(\zeta, z, \lambda)$ where $K(\zeta, z, \lambda)$ is as in Lemma 2. We obtain

$$
\int_{\partial c} f(\zeta) K(\zeta, z, \lambda)=\int_{c} d_{\zeta, \lambda}[f(\zeta) K(\zeta, z, \lambda)]=0 .
$$

The last equation in (1) follows from Lemma 2 and the fact that $f$ is holomorphic. By (1) and Lemma 1 we obtain

$$
\sum_{J} \int_{(\zeta, \lambda) \in \tau_{J} \times \Delta_{J}} f(\zeta) K(\zeta, z, \lambda)=\int_{(\zeta, \lambda) \in(\partial P) \times \Delta_{0}} f(\zeta) K(\zeta, z, \lambda)
$$

(recall that the summation $\Sigma_{J}$ is extended over all multi-indices from $\{1, \ldots, N\})$. Now notice that for $(\zeta, \lambda) \in(\partial P) \times \Delta_{0}, K(\zeta, z, \lambda)$ involves only the Bochner-Martinelli section $\gamma^{0}$. It follows from Theorem I.1 applied with the Bochner-Martinelli section $\gamma^{0}$ and the holomorphic function $f$ on the variety $V$ that

$$
\int_{(\zeta, \lambda) \in(\partial P) \times \Delta_{0}} f(\zeta) K(\zeta, z, \lambda)=c_{n, m}^{\prime} f(z)
$$

where

$$
c_{n, m}^{\prime}=(-1)^{m n}(-1)^{n(n-1) / 2+1}(2 \pi i)^{n-m}(n-m) .
$$

Now observe that for $(\zeta, \lambda) \in \tau_{J} \times \Delta_{J},(J \subset\{1, \ldots, N\})$ we have

$$
\left(\bar{\partial}_{\zeta}+d_{\lambda}\right) \gamma_{l}=d_{\lambda} \gamma_{l}
$$

and therefore for $(\zeta, \lambda) \in \tau_{J} \times \Delta_{J}$

$$
K(\zeta, z, \lambda)=\sum_{I}(-1)^{|I|} A^{I} \bigwedge_{\substack{l=1 \\ l \notin I}}^{n} d_{\lambda} \gamma_{l} \wedge \beta^{h}(\zeta)
$$

(recall that $\Sigma_{I}$ is extended over multi-indices $I=\left\{i_{0}<\cdots<i_{m}\right\} \subset$ $\{1, \ldots, n\})$. Since, by $(5), K(\zeta, z, \lambda)$ is a $(n-m-1)$-form in $d \lambda_{0}, \ldots, d \lambda_{N}$ the only terms in the sum of (2) which do not possibly vanish are those for which $J$ has exactly $(n-m)$ numbers. So (2) can be written (also in view of (3))

$$
\sum_{J^{*}} \int_{(\zeta, \lambda) \in \tau_{J^{*}} \times \Delta_{J^{*}}} f(\zeta) K(\zeta, z, \lambda)=c_{m, n}^{\prime} f(z)
$$


where $\sum_{J^{*}}$ is extended over $J^{*}=\left\{j_{1}<\cdots<j_{n-m}\right\} \subset\{1, \ldots, N\}$. All that remains to be computed now is

$$
\int_{\lambda \in \Delta_{J^{*}}} K(\zeta, z, \lambda) \quad\left(\zeta \text { restricted to } \tau_{J^{*}}\right) .
$$

So fix a $J^{*}=\left\{j_{1}<\cdots<j_{n-m}\right\} \subset\{1, \ldots, N\}$. Now the coefficient of $\eta_{s}=: \bigwedge_{k=1, k \neq s}^{n-m} d \lambda_{J_{k}}$ in $\bigwedge_{l=1, l \notin I}^{n} d_{\lambda} \gamma_{l}\left(I=\left(i_{0}<\cdots<i_{m}\right)\right)$ is

$$
B_{s}^{I}=: \frac{\partial\left(\gamma_{1}, \ldots, \hat{\gamma}_{t_{0}}, \ldots, \hat{\gamma}_{l_{1}}, \ldots, \hat{\gamma}_{i_{m}}, \ldots, \gamma_{n}\right)}{\partial\left(\lambda_{j_{1}}, \ldots, \hat{\lambda}_{J_{s}}, \ldots, \lambda_{j_{n-m}}\right)} .
$$

Therefore the "coefficient" of $\eta_{s}$ in $K(\zeta, z, \lambda)$ is

$$
\sum_{I}(-1)^{|I|} A^{I} B_{s}^{I} \beta^{h}(\zeta)
$$

which is equal to the following:

(8)

$$
\text { (8) } \begin{aligned}
& (-1)^{(m+1)(m+2) / 2} \operatorname{det}\left(H_{1}, \ldots, H_{m}, \gamma, \gamma^{J_{1}}, \ldots, \gamma^{j_{s}}, \ldots, \gamma^{j_{n-m}}\right) \beta^{h}(\zeta) \\
= & (-1)^{(m+1)(m+2) / 2} \lambda_{J_{s}} \operatorname{det}\left(H_{1}, \ldots, H_{m}, \gamma^{j_{s}}, \gamma^{j_{1}}, \ldots, \gamma^{\hat{j}_{s}}, \ldots, \gamma^{j_{n-m}}\right) \beta^{h}(\zeta) \\
= & (-1)^{(m+1)(m+2) / 2}(-1)^{s-1} \lambda_{J_{s}} \operatorname{det}\left(H_{1}, \ldots, H_{m}, \gamma^{j_{1}}, \ldots, \gamma^{J_{n-m}}\right) \beta^{h}(\zeta)
\end{aligned}
$$

(in the above determinants $\gamma$ denotes the column ${ }^{t}\left(\gamma_{1}, \ldots, \gamma_{n}\right)$ and $\gamma^{J_{k}}$ denotes the column $\left.{ }^{t}\left(\gamma_{1}^{j_{k}}, \ldots, \gamma_{n}^{J_{k}}\right)\right)$. It follows from (8) that

$$
\begin{aligned}
K(\zeta, z, \lambda)= & (-1)^{(m+1)(m+2) / 2} \operatorname{det}\left(H_{1}, \ldots, H_{m}, \gamma^{j_{1}}, \ldots, \gamma^{J_{n-m}}\right) \\
& \times \sum_{s=1}^{n-m}(-1)^{s-1} \lambda_{J_{s}} \eta_{s} \wedge \beta^{h}(\zeta)
\end{aligned}
$$

for $(\zeta, \lambda) \in \tau_{J^{*}} \times \Delta_{J^{*}}$. So in order to compute the integral (7) we need to compute

$$
\int_{\lambda \in \Delta_{J^{*}}} \sum_{s=1}^{n-m}(-1)^{s-1} \lambda_{j_{s}} \eta_{s}
$$

Recall that $\lambda \in \Delta_{J^{*}}$ means $\lambda_{J_{1}}+\cdots+\lambda_{J_{n-m}}=1$ and therefore $d \lambda_{1}=$ $-\left(d \lambda_{j_{2}}+\cdots+d \lambda_{J_{n-m}}\right)$ which substituted in $\sum_{s=1}^{n-m}(-1)^{s-1} \lambda_{j_{s}} \eta_{s}$ gives (after a computation)

$$
\sum_{s=1}^{n-m}(-1)^{s-1} \lambda_{j_{s}} \eta_{s}=d \lambda_{J_{2}} \wedge \cdots \wedge d \lambda_{j_{n-m}} \quad\left(\text { for } \lambda \in \Delta_{J^{*}}\right) .
$$

By (11) the integral (10) becomes

$$
\int_{\lambda \in \Delta_{J^{*}}} d \lambda_{J_{2}} \wedge \cdots \wedge d \lambda_{j_{n-m}}=\frac{1}{(n-m-1) !} .
$$


By (7), (12) and (9), (6) can be written

$$
f(z)=\tilde{c}_{n m} \sum_{J^{*}} \int_{\zeta \in \tau_{J^{*}}} f(\zeta) \operatorname{det}\left(H_{1}, \ldots, H_{m}, \gamma^{J_{1}}, \ldots, \gamma^{J_{n-m}}\right) \beta^{h}(\zeta)
$$

$\left(J^{*}=\left\{j_{1}<\cdots<j_{n-m}\right\} \subset\{1, \ldots, N\}\right)$ which immediately implies the integral formula of Theorem III.1 and completes the proof.

\section{REFERENCES}

[1] I. Aizenberg and A. Yuzhakov, Integral representations and residues in multidimensional complex analysis, Translation from Russian by Amer. Math. Soc., vol. 58 (1983).

[2] R. Harvey, Integral formulae connected by Dolbeault's isomorphism, Proc. of the conference on complex analysis, Rice University Studies, 56 No. 2, (1970), 67-98.

[3] T. Hatziafratis, Integral representation formulas on analytic varieties, University of Wisconsin-Madison Ph.D. Thesis (1984).

[4] G. M. Henkin, Integral representation of functions in strictly pseudoconvex domains and applications to the $\bar{\partial}$-problem, Mat. Sb., 82 (1970), 300-308, Math. USSR Sb., 11 (1970), 273-281.

[5] H. Lewy's equation and analysis on a pseudoconvex manifold I, Uspehi Mat. Nauk, 32, No. 3(195), 57-118 (1977), Russian Math. Surveys, 32 (1977), 59-130.

[6] G. Henkin and J. Leiterer, Global integral formulas for solving the $\bar{\partial}$-equation on Stein manifolds, Ann. Polonici Mathematici, XXXIX (1981), 93-116.

[7] N. Kerzman, Hölder and $L^{p}$-estimates for solutions of $\bar{\partial} u=f$ in strongly pseudoconvex domains, Comm. Pure Appl. Math., 24 (1971), 301-379.

[8] S. Krantz, Optimal Lipschitz and $L^{p}$ regularity for the equation $\bar{\partial} u=f$ on strongly pseudoconvex domains, Math. Ann., 219 (1976), 233-260.

[9] I. Lieb and R. M. Range, On integral representations and a priori Lipschitz estimates for the canonical solution of the $\bar{\partial}$-equation, Math. Ann., 265 (1983), 221-251.

[10] N. Ovrelid, Integral representation formulas and $L^{p}$-estimates for the $\bar{\partial}$-equation, Math. Scand., 29 (1971), 137-160.

[11] A. Palm, Integral representation formulas on strictly pseudoconvex domains in Stein manifolds, Duke Math. J., 43 (1976), 41-62.

[12] R. M. Range and Y. T. Siu, Uniform estimates for the $\bar{\partial}$-equation on domains with piecewise smooth strictly pseudoconvex boundaries, Math. Ann., 206 (1973), 325-354.

[13] W. Rudin, Function Theory in the Unit Ball of $\mathbf{C}^{n}$, Springer-Verlag, New York, 1980.

[14] F. Sommer, Über die Integralformeln in der Funktionentheorie mehrerer Komplexen Varänderlichen, Math. Ann., 125 (1952), 172-182.

[15] E. L. Stout, An integtral formula for the holomorphic functions on strictly pseudoconvex hypersurfaces, Duke Math. J., 42 (1975), 347-356.

[16] A. Weil, L'integrale de Cauchy et les fonctions des plusiers variables, Math. Ann., (1935), 178-182.

Received December 28, 1984 and in revised form June 20, 1985.

Syrou 21, Holargos, Attikis

155-62 GREECE 



\section{PACIFIC JOURNAL OF MATHEMATICS EDITORS}

V. S. VARADARAJAN

(Managing Editor)

University of California

Los Angeles, CA 90024

HERBERT CLEMENS

University of Utah

Salt Lake City, UT 84112

R. FINN

Stanford University

Stanford, CA 94305
HERMANN FLASCHKA

University of Arizona

Tucson, AZ 85721

RAMESH A. GANGOLLI

University of Washington

Seattle, WA 98195

VAUGHAN F. R. JONES

University of California

Berkeley, CA 94720

ROBION KIRBY

University of California

Berkeley, CA 94720
C. C. MOORE

University of California

Berkeley, CA 94720

H. SAMELSON

Stanford University

Stanford, CA 94305

HAROLD STARK

University of California, San Diego

La Jolla, CA 92093

\section{ASSOCIATE EDITORS}

R. ARENS

E. F. BECKENBACH

B. H. NEUMANN (1906-1982)
F. WOLF

K. YOSHIDA

\section{SUPPORTING INSTITUTIONS}

UNIVERSITY OF ARIZONA

UNIVERSITY OF BRITISH COLUMBIA

CALIFORNIA INSTITUTE OF TECHNOLOGY

UNIVERSITY OF CALIFORNIA

MONTANA STATE UNIVERSITY

UNIVERSITY OF NEVADA, RENO

NEW MEXICO STATE UNIVERSITY

OREGON STATE UNIVERSITY
UNIVERSITY OF OREGON UNIVERSITY OF SOUTHERN CALIFORNIA

STANFORD UNIVERSITY

UNIVERSITY OF HAWAII

UNIVERSITY OF TOKYO

UNIVERSITY OF UTAH

WASHINGTON STATE UNIVERSITY

UNIVERSITY OF WASHINGTON 


\section{Pacific Journal of Mathematics}

\section{Vol. 123, No. $1 \quad$ March, 1986}

Maria Emilia Alonso García, A note on orderings on algebraic varieties $\ldots \ldots 1$

F. S. De Blasi and Józef Myjak, On continuous approximations for

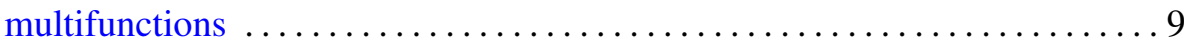

Frank Albert Farris, An intrinsic construction of Fefferman's CR metric . . . 33 Antonio Giambruno, P. Misso and Francisco César Polcino Milies, Derivations with invertible values in rings with involution $\ldots . \ldots . \ldots .47$

Dan Haran and Moshe Jarden, The absolute Galois group of a pseudo real

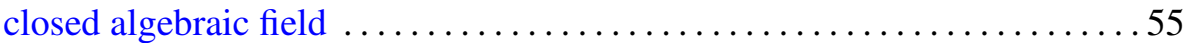

Telemachos E. Hatziafratis, Integral representation formulas on analytic

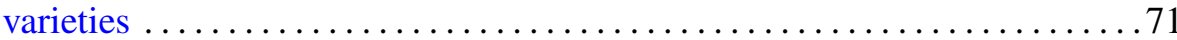

Douglas Austin Hensley, Dirichlet's theorem for the ring of polynomials

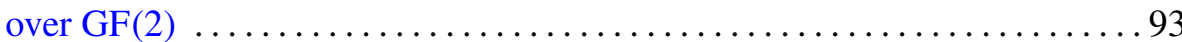

Sofia Kalpazidou, On a problem of Gauss-Kuzmin type for continued

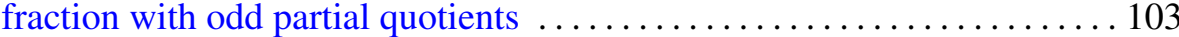

Harvey Bayard Keynes and Mahesh Nerurkar, Ergodicity in affine

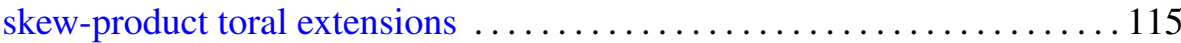

Thomas Landes, Normal structure and the sum-property $\ldots \ldots \ldots \ldots \ldots 127$

Anthony To-Ming Lau and Viktor Losert, Weak*-closed complemented invariant subspaces of $L_{\infty}(G)$ and amenable locally compact groups ...149 Andrew Lelek, Continua of constant distances in span theory . . . . . . . . 161 Dominikus Noll, Sums and products of $B_{r}$ spaces $\ldots \ldots \ldots \ldots \ldots \ldots \ldots \ldots$ Lucimar Nova, Fixed point theorems for some discontinuous operators 189

A. A. S. Perera and Donald Rayl Wilken, On extreme points and support points of the family of starlike functions of order $\alpha$

Massimo A. Picardello, Positive definite functions and $L^{p}$ convolution operators on amalgams ........................

Friedrich Roesler, Squarefree integers in nonlinear sequences ......... 223

Theodore Shifrin, The osculatory behavior of surfaces in $\mathbf{P}^{5}$ 